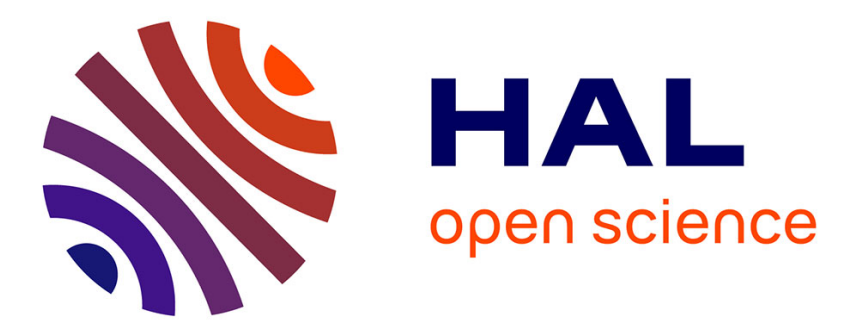

\title{
Pagnes partisans, fabrique des partis et participation politique des femmes des classes populaires au Malawi \\ Paul Grassin
}

\section{To cite this version:}

Paul Grassin. Pagnes partisans, fabrique des partis et participation politique des femmes des classes populaires au Malawi. Politique africaine, 2009. hal-01646824

\section{HAL Id: hal-01646824 \\ https://hal.science/hal-01646824}

Submitted on 23 Nov 2017

HAL is a multi-disciplinary open access archive for the deposit and dissemination of scientific research documents, whether they are published or not. The documents may come from teaching and research institutions in France or abroad, or from public or private research centers.
L'archive ouverte pluridisciplinaire HAL, est destinée au dépôt et à la diffusion de documents scientifiques de niveau recherche, publiés ou non, émanant des établissements d'enseignement et de recherche français ou étrangers, des laboratoires publics ou privés. 
Paul Grassin

\title{
Pagnes partisans, fabrique des partis et participation politique des femmes des classes populaires au Malawi
}

\begin{abstract}
Au Malawi, les tissus politiques arborant les couleurs et symboles des partis sont omniprésents dans l'espace public en période électorale, mais également en dehors, au quotidien, allant jusqu'à pénétrer les espaces domestiques. Cet article considère les usages du tissu partisan par les élites politiques, les militants et les simples citoyens comme un moyen d'éclairer la fabrique des partis et leur ancrage social, ainsi que les différents modes de politisation et de participation politique des femmes des catégories populaires.
\end{abstract}

Élément central de la vie politique après l'indépendance en 1964, le pagne (chitenje, pluriel : zitenje) partisan - représentant les couleurs, les symboles du parti et le portrait de son leader est aujourd'hui devenu un objet incontournable du rituel électoral au Malawi ${ }^{1}$. Généralement distribué lors des meetings de campagne, cet habit féminin est habituellement noué autour de la taille ou, de manière plus sophistiquée, porté en national suit lors de ces mêmes meetings ${ }^{2}$. Ainsi vêtues, les femmes s'engagent dans des chants et des danses collectives. En période de campagne, le pagne envahit l'ensemble du paysage : il habille les minibus qui sillonnent le territoire, décore les tribunes officielles, flotte en étendard à la cime des arbres... Mais, une fois le processus électoral achevé, il continue à circuler au quotidien et à occuper les espaces publics et domestiques, porté comme vêtement dans la vie de tous les jours, dans les champs et les marchés, mais aussi, plus singulièrement, utilisé comme linge de maison, comme rideaux ou pour transporter des marchandises. Le pagne n'est pas l'unique vêtement partisan à être distribué de la sorte et à circuler dans l'espace public malawien ${ }^{3}$, mais il est particulièrement intéressant car exclusivement féminin, et permet donc de mettre en lumière la participation spécifique des femmes à la vie politique et partisane ${ }^{4}$.

Si des femmes des classes sociales supérieures, appartenant aux cercles dirigeants des partis, portent le pagne dans les meetings politiques, les usages quotidiens sont quant à eux l'apanage des catégories populaires. Alors que l'analyse de la participation politique des femmes malawiennes s'est jusqu'à présent très unilatéralement focalisée sur les danses collectives dans les meetings de campagne ${ }^{5}$, l'approche par le pagne et ses usages permet de dépasser le cadre des élections et des instances partisanes pour étudier la politisation des femmes des catégories populaires dans sa dimension quotidienne. Cela implique d'observer les usages qui sont faits de ces tissus sans se limiter au calendrier électoral et de s'interroger, ce faisant, sur le devenir

1 . Je suis très reconnaissant envers Florence Brisset-Foucault pour sa lecture exigeante et son aide généreusement apportée à la finalisation de cet article et envers les relecteurs anonymes de Politique africaine dont les nombreuses remarques ont très largement contribué à son amélioration.

2 . Le national suit est un ensemble complet comportant une jupe, un haut et un couvre-chef. Il est devenu, depuis l'indépendance, l'habit politique usuel des femmes.

3 . Quelques cadres nationaux masculins peuvent être amenés à porter des chemises confectionnées avec le pagne du parti, mais cet usage reste limité aux élites partisanes et dans le strict cadre des meetings.

4 . On trouve également des T-shirts affichant une symbolique similaire et portés indifféremment par les hommes et par les femmes.

5 . Voir L. Gilman, The Dance of Politics: Gender, Performance and Democratization in Malawi, Philadelphie, Temple University Press, 2009 ; E. L. Mkamanga, Suffering in Silence: Malawi Women's 30 Year Dance with Dr Banda, Glasgow, Dudu Nsomba Publications, 2000. 


\section{DERNIÈRE VERSION AVANT PUBLICATION}

social de ces objets. À travers l'analyse des trajectoires biographiques de ces tissus ${ }^{6}$, des pratiques et des perceptions des femmes qui les utilisent, cet article s'attache à montrer que des rapports variés à l'engagement politique et à l'adhésion partisane sont contenus dans les différents usages des zitenje ${ }^{7}$.

Enfin, cet article considère les conditions de distribution et d'usage du pagne comme un élément central de la «fabrique des organisations partisanes ${ }^{8}$ », révélateur de leur genèse et de leurs modes de fonctionnements internes, mais aussi des modalités de leur ancrage social et territorial. L'ensemble des partis politiques malawiens investissent temps et argent dans la confection et la distribution des pièces de tissu imprimé. Ce dernier offre alors un point de vue privilégié sur le rôle des femmes dans ce processus. Le propos s'articule autour de l'idée que l'imagerie et la rhétorique militantes investissent l'espace public par l'intermédiaire privilégié des corps utilisés comme «infrastructures partisanes ${ }^{9}$ ». On attend des porteuses qu'elles fassent exister le parti au quotidien dans l'espace public et qu'elles en fassent la publicité. Toutefois, plutôt que d'expliquer le recours massif aux tissus par la simple absence d'infrastructures alternatives, il s'agit ici de rendre compte de l'épaisseur historique et sociale des usages politiques du chitenje en les replaçant dans l'histoire politique postcoloniale et dans le cadre plus large des pratiques vestimentaires locales ${ }^{10}$.

Alors que le Malawi Congress Party (MCP), au pouvoir après l'indépendance, faisait payer aux membres l'acquisition des pagnes, les nouveaux partis issus du multipartisme se sont mis à distribuer massivement et gratuitement ces pièces de tissu dans les meetings de campagne ${ }^{11}$. Par conséquent, toutes les femmes qui le portent au quotidien ne sont pas nécessairement membres, ni même supportrices du parti dont elles arborent les couleurs (on verra qu'il en est parfois de même pour les danseuses des meetings politiques). Ces usages non militants, dans des espaces domestiques ou des activités a priori exclues du champ politique officiel, soulèvent

. I. Kopytoff, « The Cultural Biography of Things: Commoditization as Process », in A. Appadurai (dir.),

The Social Life of Things: Commodities in Cultural Perspective, Cambridge, Cambridge University Press, 1986, p. 64-91.

7 . L'article présenté ici est le résultat d'une enquête ethnographique réalisée en trois phases. À l'observation des meetings de campagne en zone rurale, réalisée en amont des élections tripartites de 2014, s'ajoutent des entretiens menés courant 2015 auprès d'élus locaux dans les quartiers populaires de Blantyre, puis en 2016 dans trois marchés de l'agglomération de Blantyre auprès des femmes qui les utilisent. Le fait de vivre au quotidien dans les espaces sociaux étudiés m'a par ailleurs donné l'occasion de mesurer les circulations et les usages des vêtements en dehors des arènes politiques officielles jusqu'au cœur des espaces domestiques.

8 . H. Combes, Faire parti : trajectoires de gauche au Mexique, Paris, Karthala, coll. « Recherches internationales », 2011.

9 . Je m'appuie ici sur la notion de «people as infrastructure » proposée par AbouMaliq Simone pour désigner le rôle des personnes, de leurs corps et de leurs activités comme support pour la circulation des biens et du pouvoir dans le centre-ville de Johannesburg. Voir A. Simone, «People as Infrastructure: Intersecting Fragments in Johannesburg », Public Culture, vol. 16, n 3, 2004, p. 407-429.

10 . Le rôle central des panoplies partisanes dans les campagnes électorales ne se limite d'ailleurs ni au cas malawien, ni au continent africain, mais s'observe également dans des contextes, comme les États-Unis d'Amérique, où de grandes infrastructures existent.

11 . Malgré la transition, le MCP continue de faire payer l'accès à ses pagnes, les femmes qui veulent en obtenir doivent les acheter. 


\section{DERNIÈRE VERSION AVANT PUBLICATION}

la question de l'évolution du statut et du sens politique donné à cet objet. Par ailleurs, l'enjeu pour les membres est de parvenir à se distinguer de ces usages détournés, une distinction rendue d'autant plus complexe qu'usages militants et non militants peuvent se recouper et coexister dans les mêmes espaces. Bons et mauvais usages du pagne se retrouvent alors au cœur de luttes morales pour la définition des pratiques militantes féminines.

Lorsqu'ils le distribuent à leurs membres à travers les structures locales du parti ou aux électeurs lors des meetings de campagne, les élites politiques donnent pour unique consigne aux femmes de le porter lors des meetings. Toute autre utilisation quotidienne est donc le fruit d'une démarche personnelle. En occupant une position stratégique à «l'interface entre l'individu et le monde social ${ }^{12} »$, le vêtement partisan fonctionne comme un puissant vecteur d'identification et de participation politique pour les femmes.

En tant qu'objet intime et utilisable au quotidien, dans une grande diversité de contextes, il leur offre des marges de manœuvre pour définir et mettre en pratique leur militantisme, cela même dans le cadre très ritualisé des meetings. On soutient ici que c'est à travers ces utilisations personnelles du pagne que se négocie la participation des femmes à la politique partisane et à la compétition électorale ${ }^{13}$. Dans un mouvement inverse, nous verrons qu'en connectant directement le parti avec les corps des femmes qui le porte, il fonctionne comme un outil privilégié de contrôle et d'encadrement du travail militant.

Éclairer le fait partisan et le processus électoral par le tissu suppose de se situer à la convergence de trois littératures différentes : la sociologie des réseaux militants ${ }^{14}$, celle des organisations et des milieux partisans ${ }^{15}$, ainsi que les études sur le rôle des objets dans les processus de subjectivation ${ }^{16}$. L'approche par les réseaux, qui constituent l'assise sociale des partis, répond à une volonté d'éclairer les modes d'investissement ou de désinvestissement de la politique partisane par les classes populaires. On cherche par-là à renouveler l'étude des partis politiques au Malawi, jusqu'à présent unilatéralement centrée sur les circulations, les coups et les stratégies de leurs élites dirigeantes ${ }^{17}$. En s'intéressant à la manière dont les individus vivent

. J. M. Allman, Fashioning Africa: Power and the Politics of Dress, Bloomington/Indianapolis, Indiana University Press, 2004, p. 1-2.

13 . Des conclusions similaires concernant la négociation de la loyauté des femmes au régime camerounais sont établies par M.-E. Pommerolle et N. M. Ngaméni, «Fabrics of Loyalty: The Politics of International Women's Day Wax Print Cloth in Cameroon », Africa, vol. 85, n 4, 2015, p. 656-676.

14 . S. G. Tarrow, Power in Movement: Social Movements, Collective Action and Politics, Cambridge, Cambridge University Press, coll. « Cambridge Studies in Comparative Politics », 1994 ; M. Diani et D. McAdam (dir.), Social Movements and Networks: Relational Approaches to Collective Action, Oxford, Oxford University Press, coll. « Comparative Politics », 2003.

15 . F. Sawicki désigne ainsi « l'ensemble des individus et des groupes - ainsi que les réseaux qui les lient -, dont les activités contribuent, sans que cet objectif soit nécessairement visé, à faire exister un parti donné ». Voir F. Sawicki, « Les partis politiques comme entreprises culturelles », in D. Cefaï (dir.), Les cultures politiques, Paris, PUF, coll. « Politiques d'aujourd'hui », 2001, p. 191-212.

16 . J.-P. Warnier, «Pour une praxéologie de la subjectivation politique », in J.-F. Bayart et J.-P. Warnier (dir.), Matière à politique : le pouvoir, les corps et les choses, Paris, Karthala, coll. « Recherches internationales », 2004, p. 7-31.

17 . L. Rakner, L. Svåsand et N. S. Khembo, «Fissions and Fusions, Foes and Friends Party System Restructuring in Malawi in the 2004 General Elections », Comparative Political Studies, vol. 40, n 9, 2007 , 


\section{DERNIÈRE VERSION AVANT PUBLICATION}

leur participation au jeu politique, aux différentes formes de rétribution qu'elle suscite, ainsi qu'aux interactions entre gouvernants et gouvernés que la circulation et les usages du tissu partisan impliquent, on cherchera également à se distinguer des analyses communément admises sur la dimension purement utilitaire et manipulatrice de la relation entre les patrons politiques et leurs clients ${ }^{18}$.

Le propos s'articule autour de trois axes. Le premier étudie la contribution du pagne à la fabrique partisane : à la production de sa structure interne d'une part et à la formation de son idéologie politique d'autre part. Nous verrons notamment comment rapports hiérarchiques et division sociale et sexuelle du travail militant sont d'autant plus marqués qu'ils sont directement visibles sur les corps à travers le vêtement. Le second axe revient sur les évolutions des modes de distribution du pagne suite à l'introduction du multipartisme et leurs conséquences sur la diversification de ses usages quotidiens par les membres, mais également par des femmes nonmembres. Nous explorerons les différents rapports à l'engagement contenus dans ces pratiques et nous verrons la façon dont « bons » et « mauvais » usages participent au quotidien à l'ancrage partisan d'une part et à la compétition politique de l'autre. La dernière partie de ce travail s'intéressera plus précisément aux usages personnels que font les femmes membres des partis de leurs pagnes et aux registres moraux qu'elles invoquent pour se distinguer des usages catégorisés comme non militants. Nous montrerons alors que l'utilisation par les élites partisanes des femmes habillées pour leurs batailles politiques tient en grande partie au fait que le chitenje, et la liberté qu'elles ont de s'en servir comme de le tailler, leur permet de définir et de contrôler elles-mêmes les formes quotidiennes de leur militantisme.

\section{L'habit et la fabrique partisane}

En cours d'entretien, Maria Nkholokossa, responsable de la branche des jeunes du Democratic Progressive Party (DPP) dans le quartier populaire de Ndirande à Blantyre, dont elle est membre depuis sa création, part dans sa maison et revient avec une pile de pagnes et de T-shirts du parti soigneusement pliés qu'elle me présente un à un :

«Celui-ci, c'est un T-shirt donné par le maire du quartier (councillor) pendant sa campagne. Je le porte encore quand le maire organise une réunion pour lui montrer ma reconnaissance. Celuici date de celle de 2009 de Bingu Mutharika et, celui-ci, Peter Mutharika me l'a donné en personne pendant un meeting électoral en 2014. Ceux-là [elle montre le T-shirt de Peter [Mutharika] et le national suit qu'elle a fait tailler avec le pagne] sont mes préférés. Je ne les porte que pour les meetings ${ }^{19}{ }$.

Comme l'illustrent ces propos d'une membre locale du parti au pouvoir, le chitenje joue un rôle tout à fait central dans la personnalisation des liens politiques verticaux qui structurent les

p. $1112-1137$; L. Rakner et L. Svåsand, «Competition and Participation, but No Democracy: The Politics of Elections in Africa's Feckless Regimes », Statsvetenskaplig Tidskrift, vol. 115, n 4, 2013.

18 . D. Gaxie, «Économie des partis et rétributions du militantisme », Revue française de science politique, vol. 27, $\mathrm{n}^{\circ}$ 1, 1977, p. 123-154; voir également H. Combes et G. Vommaro, «Relations clientélaires ou politisation : pour dépasser certaines limites de l'étude du clientélisme », Cahiers des Amériques latines, ${ }^{\circ} 69$, 2012, p. 17-35. 


\section{DERNIÈRE VERSION AVANT PUBLICATION}

organisations partisanes et la vie politique au Malawi ${ }^{20}$. L'aspect fortement ritualisé de la distribution des pagnes - remis gratuitement et en personne aux femmes par les candidats ou les cadres du parti en amont ou en plein cœur des meetings - renforce cet aspect ${ }^{21}$. Cette mise en scène du don a pour objectif de démontrer la générosité et la richesse du leader, en plus d'établir un rapport direct avec les électrices. L'ensemble des femmes rencontrées se souviennent ainsi de la personne qui leur a remis chaque pagne ou T-shirt qu'elles possèdent. Par ailleurs, le tissu affiche généralement le portrait du leader à l'origine de la création du parti, renforçant par là la dimension patrimoniale des rapports politiques. Le choix des moments et des façons de porter le vêtement est ainsi un moyen d'affirmer leur rapport personnel avec celui ou celle qui le leur a offert et le lien politique paternaliste qui les unit au fondateur ou à la fondatrice du parti. Cette fonction politique du vêtement remonte au « populisme culturel ${ }^{22}$ »et à l'entreprise d'africanisation du régime de parti unique dirigé par le $\mathrm{D}^{\mathrm{r}} \mathrm{Kamuzu}$ Banda immédiatement après l'indépendance ${ }^{23}$. Les pagnes servaient d'une part à l'exposition des symboles et des valeurs du régime et d'autre part comme outil d'encadrement de la participation politique des femmes ${ }^{24}$. Le tissu aux couleurs du parti unique et à l'effigie du dictateur symbolisait alors le lien paternaliste entre le leader national et les femmes du pays ${ }^{25}$. À chaque meeting ou cérémonie officielle présidé par le président à vie, des femmes sélectionnées au sein de la branche féminine locale du parti étaient tenues de danser et chanter à son honneur, habillées avec le chitenje du parti. Pour qualifier la relation qui l'unit à ces danseuses, Banda s'appuyaient sur une relecture nationaliste des concepts chewa de Nkhoswe et de Mbumba, désignant respectivement le leader familial et le groupe composé de ses sœurs et de leurs filles placées sous son autorité ${ }^{26}$; par extension, toutes les femmes du Malawi portant le vêtement du MCP étaient symboliquement incluses dans ce lien paternaliste avec le leader.

Mais, au-delà de leur rôle dans la personnalisation des rapports verticaux, les pagnes contribuent également à la production de rapports horizontaux entre les membres des partis. En analysant la façon dont le tissu imprimé s'est historiquement imposé comme un bien de consommation et d'échange central au Malawi - porté et utilisé dans une grande variété de contextes sociaux, religieux et politiques -, Sarah Worden montre comment celui-ci a joué un rôle de premier plan dans la formation des identités sociales des femmes ${ }^{27}$. Les entretiens réalisés auprès des commerçantes dans trois marchés de l'agglomération de Blantyre tendent à

. D. Cammack, «The Politics of Chameleons Revisited: The Burden of Malawi’s Political Culture », in

M. Ott et E. Kanyongolo (dir.), Democracy in Progress: Malawi's 2009 Parliamentary and Presidential Elections, Balaka, Kachere Series, 2010, p. 153-184.

. L. Gilman, The Dance of Politics..., op. cit., p. 115.

. R. M. Chirambo, «Democracy As Limiting Factor For Politicised Cultural Populism in Malawi »,

Africa Spectrum, vol. 44, n 2, 2009, p. 77-94.

23 . S. Worden, « Chitenje: The Production and Use of Printed Cotton Cloth in Malawi », Textile Society of America Symposium Proceedings, septembre 2014, <http://digitalcommons.unl.edu/tsaconf/888> ; P. Faber, Long Live the President: Portrait-Cloths from Africa, vol. 1, Amsterdam, KIT Publishers, 2010.

24 . S. Worden, « The Chitenje: Dress and Politics in Malawi », European Conference of African Studies, Paris, juillet 2015.

25 . L. Gilman, The Dance of Politics..., op. cit., p. 43.

26 .W. C. Chirwa, «Dancing Towards Dictatorship: Political Songs and Popular Culture in Malawi », Nordic Journal of African Studies, vol. 10, $\mathrm{n}^{\circ}$ 1, 2001, p. 1-27. 


\section{DERNIÈRE VERSION AVANT PUBLICATION}

montrer que les pagnes partisans, portés au quotidien, hors période électorale, fonctionnent de la même façon comme un outil d'identification et de reconnaissance mutuelle entre les membres du parti. Il fait office de carte d'adhésion et procure le sentiment d'appartenir à un groupe de pairs. C'est ce qui ressort des propos de Maria Nkholokossa lorsque je lui demande pourquoi les femmes les portent dans les marchés et les espaces publics :

«Si on le porte autour de la taille, c'est pour s'identifier, pour afficher notre appartenance au parti [...] et pour être reconnue par les autres aussi. Par exemple si vous avez un accident, les gens vont demander comment vous étiez habillée, et là ils sauront que vous appartenez au parti. D'autres membres qui sont là pourront vous porter assistance ${ }^{28} »$.

\section{Le pagne comme support doctrinal}

Se doter d'une identité visuelle transposée sur du tissu est un enjeu primordial pour toute organisation partisane au Malawi et constitue l'une des premières tâches que celle-ci accomplit dès sa création. Chaque pagne contient ce que l'on pourrait qualifier d'ADN idéologique du parti, c'est-à-dire les éléments qui ont contribué à sa formation. L'étude de la genèse des partis au Malawi est souvent réduite aux stratégies carriéristes de leurs fondateurs et véhicule l'image d'un système partisan idéologiquement indifférencié ${ }^{29}$. Une des critiques les plus récurrentes porte sur leur utilisation uniforme, abusive, populiste et opportuniste du lexique du développement ${ }^{30}$. L'analyse du contenu symbolique des pagnes indique pourtant des différences notables dans leur interprétation du concept, entre une vision plus politique et une autre davantage socio-économique. Dans un pays où liberté politique et prospérité économique ont historiquement peiné à coexister, ces différences idéologiques ne doivent pas être sousestimées. Les symboles présents sur les tissus du Democratic Progressive Party (DPP) et du People's Party (PP), respectivement les deux derniers au pouvoir, illustrent parfaitement ces écarts. Aux quatre épis de maïs couronnant le nom du DPP, s'oppose un cadenas ouvert avec sa clef pour le PP. Avec le choix du maïs, première denrée produite dans le pays, base de l'alimentation et véritable institution sociale en zone rurale, le DPP renoue avec une lecture ruraliste, agricole et productiviste du développement, qui avait été celle du Docteur Banda et dont le régime précédant la naissance du parti en 2004 s'était distancié ${ }^{31}$. En associant ainsi le développement à la sécurité alimentaire et à la production agricole, le DPP s'adresse à un électorat paysan, durement touché par la libéralisation des marchés agricoles et par la famine ayant sévi les années auparavant. Le développementalisme paysan, tel qu'il est affiché sur le tissu, renvoie également à la nécessité de se démarquer idéologiquement de l'ancien régime. La même remarque peut être faite concernant le parti pris idéologique du PP au moment de sa création en 2012 suite à une scission du DPP au pouvoir, alors que celui-ci est attaqué de toutes parts pour la répression sanglante des manifestations de juillet 2011 et pour sa dérive autoritaire.

28 . Entretien avec Maria Nkholokossa, Ndirande, 23 octobre 2016.

29 . L. Rakner, L. Svåsand et N. S. Khembo, «Fissions and Fusions... », art. cité. Pour une remise en cause de cette idée à partir d'une étude des manifestes des partis, voir A. M. Mpesi, «Political Parties and their Manifestos: Inferring Party Policy Positions in Malawi since 1994 », Forum for Development Studies, vol. 38, $\mathrm{n}^{\circ} 1,2011$, p. $25-41$.

30 . H. Englund, Human Rights and African Airwaves: Mediating Equality on the Chichewa Radio, Bloomington, Indiana University Press, 2011.

31 . E. C Mandala, The End of Chidyerano: A History of Food and Everyday Life in Malawi, 1860-2004, Portsmouth, Heinemann, 2005. 


\section{DERNIÈRE VERSION AVANT PUBLICATION}

Les fondateurs cherchent à se différencier de leur ancienne famille politique en proposant une vision plus institutionnelle et plus politique du développement basée sur la transparence, l'ouverture des institutions politiques au peuple et la garantie des libertés politiques, représentées sur le tissu du PP par le cadenas ouvert ${ }^{32}$.

\section{Le pagne et la division du travail partisan}

La place de l'habit dans la fabrique partisane tient enfin au rôle central qu'il joue dans la production de la structure du parti et dans l'identification des positions dans sa hiérarchie. Les meetings, particulièrement nombreux en période électorale, constituent un cadre empirique privilégié pour appréhender ce rôle ${ }^{33}$. Moments festifs et fortement ritualisés, ils réunissent en un même lieu élites partisanes, militants et électeurs. Un regard attentif aux différentes façons de porter les vêtements du parti lors de ces événements nous informe alors sur la division du travail militant et sur les rapports de domination qui traversent les organisations politiques.

Quel que soit le meeting observé, toute personne prenant part aux activités et aux prises de parole porte le vêtement partisan, à l'exception des groupes artistiques locaux dont les performances ponctuent les cérémonies. Dans l'audience, sa présence est bien plus faible et prend des significations différentes sur lesquelles nous reviendrons plus en aval. Tout se passe donc comme si le port de ce vêtement agissait comme un sésame pour participer, en conférant aux agents la légitimité à intervenir dans le meeting.

Une observation fine de l'habit permet d'identifier trois types de membres ainsi que les rôles assignés à chacun d'eux : les cadres dirigeants masculins, les femmes et les jeunes hommes ${ }^{34}$. Quel que soit le meeting observé, les cadres masculins, tous installés en tribune, sont les seuls à prendre la parole au micro. On les reconnaît à leur costume, dont la chemise est parfois confectionnée avec le pagne du parti ou, plus sobrement, dont la cravate est à ses couleurs (figure 1). Les membres de l'aile jeunesse (youth wing) sont identifiables au fait qu'ils portent le T-shirt du parti. Ils ne prennent pas part aux discussions mais sont chargés d'assurer la sécurité et la bonne tenue du meeting, en plus de gérer la sono et de jouer du tambour pour accompagner les danses des femmes. Les plus nombreuses sont les femmes. Vêtues du pagne, elles sont présentes sur les lieux bien en amont du meeting, non seulement pour accueillir les intervenants, mais également pour annoncer la tenue du meeting et mobiliser les habitants des environs. Au cours de l'événement, elles sont assises par terre au premier rang, face à la tribune officielle et ponctuent les interventions des orateurs par des danses et des chants glorifiant le parti et les leaders présents ${ }^{35}$.

32 . Entretien avec une membre de l'encadrement du PP, Blantyre, 26 octobre 2016.

33 . Pour une réflexion sur la façon dont les meetings informent sur le fonctionnement interne des partis, voir H. Combes, «Meetings de fin de campagne au Mexique et ethnographie des milieux partisans », Politix, $\mathrm{n}^{\circ} 85,2009$, p. $149-179$.

34 . Calqués sur le modèle du MCP dès l'indépendance, les partis politiques sont tous composés de trois branches: une branche centrale composée des hommes, une branche féminine et une branche jeune. Chaque branche comprend une structure autonome du niveau local au niveau national.

35 . Pour une étude détaillée de ces danses, voir L. Gilman, The Dance of Politics..., op. cit. 


\section{DERNIÈRE VERSION AVANT PUBLICATION}

Des écarts importants existent dans les façons de porter le pagne entre celles qui le taillent en vêtement et celles qui se contentent de le nouer autour de la taille. Ces différences traduisent des écarts de position dans la hiérarchie. En effet, si le tissu est distribué massivement et gratuitement, il reste très rare pour une militante qui n'occupe pas de position au moins à l'échelle du district d'en posséder plus d'une pièce. Or la taille d'un habit en nécessite au moins deux, voire trois, pour la confection d'un national suit complet. Ann Kan'gombe, qui n'est que secrétaire d'une branche locale au nord de Blantyre, m'avoue ainsi ne pas avoir, du fait de sa position, les connexions nécessaires pour acquérir une pièce de plus que celle qu'elle a reçue gratuitement des mains du candidat au poste de député lors de la campagne de $2014^{36}$. À l'inverse, une position permettant un accès direct aux élus ou aux cadres régionaux donne également un accès privilégié au tissu. Ainsi, en plus de conférer une identité collective, le chitenje inscrit la structure hiérarchique du parti sur les corps de ses membres; il hiérarchise les interventions et participe à la division sociale et sexuelle du travail militant entre aînés et cadets.

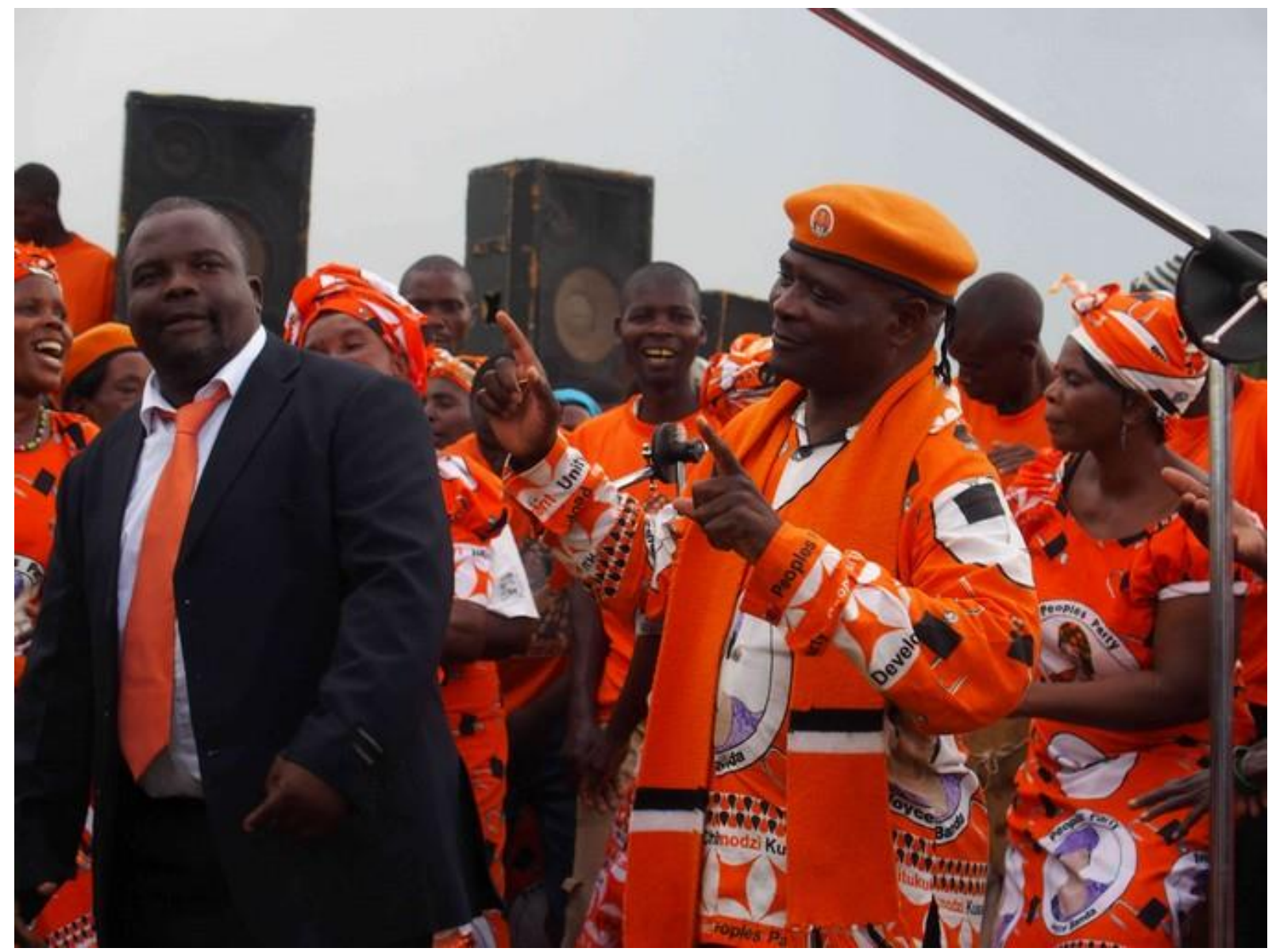

Illustration 1 : à droite, le maître de cérémonie en costume partisan lors du meeting du PP, 16 mars 2014 


\section{DERNIÈRE VERSION AVANT PUBLICATION}

\section{« Bons » et « mauvais » usages du pagne dans la compétition partisane}

L'introduction du multipartisme en 1994 a profondément transformé les enjeux associés à la distribution et aux usages du pagne partisan : ce n'est plus seulement la mise en scène de la loyauté des femmes au parti et au régime qui est en jeu, mais surtout le besoin pour les nouveaux partis de s'ancrer sur un territoire où la population est majoritairement rurale et où les infrastructures physiques sont rares. Alors que le MCP de l'époque du parti unique distribuait ses zitenje au compte-gouttes à travers ses structures locales et faisait payer les militantes, ceux des nouveaux partis sont distribués massivement et gratuitement lors des meetings. En période électorale, T-shirts, chapeaux, parapluies et autres objets aux couleurs des partis sont également importés de Chine par containers et ainsi distribués aux côtés des pagnes qui sont, eux, confectionnés par l'usine Mapeto à Blantyre ${ }^{37}$. Comme me l'explique un cadre du People's Party, l'objectif est de se rendre visible : «Pour nous il est important que les gens le portent, cela signifie que nous sommes visibles, que nous ne sommes pas morts [...] si les gens portent encore nos couleurs ${ }^{38} »$. Pour des partis politiques dont les ressources financières sont très limitées, le chitenje - au tissu très fin et aux teintures de basse qualité - représente un outil de communication peu coûteux ${ }^{39}$.

Avant d'être un uniforme utilisé par les partis - mais aussi par des églises, des ONG et d'autres organisations culturelles -, c'est d'abord un vêtement historiquement porté par l'ensemble des femmes malawiennes, représentant encore aujourd'hui un marché massif ${ }^{40}$. Si la mode occidentale est aujourd'hui particulièrement suivie, notamment en zone urbaine par les jeunes femmes des classes moyennes, les femmes des quartiers populaires et des zones rurales continuent à nouer un pagne autour de leur taille au quotidien et sur leur lieu de travail. Il s'agit d'une pratique vestimentaire apprise et transmise entre les générations au sein de la famille. À l'adolescence, on apprend aux femmes à porter ces pièces de tissus autour de leur taille lorsqu'elles sont en public pour masquer leurs jambes ${ }^{41}$. Les organisations partisanes s'appuient directement sur ces pratiques vestimentaires locales pour s'ancrer sur le territoire. Elles accordent leurs stratégies de distribution sur les évolutions de la mode et les usages sélectifs du pagne :

Paul Grassin : Pourquoi vous appuyez-vous sur le chitenje ?

Membre de l'encadrement du PP : Parce que les femmes les portent, c'est notre culture.

P. G. : Et les femmes sont importantes en termes de communication politique ?

PP : Les femmes sont celles qui viennent aux meetings politiques [...]. Mais elles sont aussi celles que l'on voit dans l'espace public. Les femmes et les jeunes. Mais les jeunes ne portent pas les zitenje $[\ldots]$ alors nous avons des T-shirts.

P. G. : Les femmes les portent partout, au marché, au travail...

PP : Sur le marché oui, mais pas dans les bureaux. C'est pourquoi nous avons tendance à cibler les femmes qui vendent sur les marchés quand on distribue le tissu ${ }^{42}$.

37 . S. Worden, « Chitenje: The Production... », art. cité.

38 . Entretien avec une membre de l'encadrement du PP, Blantyre, 26 octobre 2016.

39 . Tous s'approvisionnent chez le même fabricant, l'usine Mapeto à Blantyre.

$40 \quad$. S. Worden, « Chitenje: The Production... », art. cité.

41. Entretien avec Ann Kan'gombe, halles de Blantyre, 24 octobre 2016.

42 . Entretien avec une membre de l'encadrement du PP, Blantyre, 26 octobre 2016. 


\section{DERNIÈRE VERSION AVANT PUBLICATION}

C'est ce qui explique que l'on retrouve l'habit partisan surtout dans les marchés, porté par les vendeuses de légumes et de produits agricoles. L'introduction de plus en plus massive des Tshirts s'inscrit quant à elle dans un processus de modernisation des partis et répond à un besoin d'exister au quotidien sur le corps des jeunes hommes - très présents dans les marchés et les gares routières - en plus de celui des femmes.

Ainsi, le tissu partisan joue un rôle décisif dans la compétition politique par l'image. En parvenant à exister sur les corps des individus, les partis montrent non seulement qu'ils existent, mais affichent également leur capacité mobilisatrice : «Ça montre que nous sommes vivants et que l'on a des gens », continue mon interlocutrice du PP.

Le lancement de la campagne du United Democratic Front (UDF) en 2014, en « envahissant les rues de Blantyre d'une marée jaune ${ }^{43} \gg-$ la couleur du parti -, est un exemple paradigmatique de ces stratégies. Tous les partis ne sont pas égaux dans cette lutte. N'ayant pas de fonds propres et dépendants des donations privées, ils font généralement reposer les frais de campagne et d'organisation des meetings sur les candidats ou les élus. Seul le parti au pouvoir, qui attire davantage de donations mais obtient également des crédits auprès du fabricant, est en mesure de fournir le matériel de campagne à ses candidats ${ }^{44}$. Avant une échéance électorale, mais aussi durant la période qui la sépare de la suivante, c'est donc les couleurs de ce parti que l'on retrouve le plus massivement dans l'espace public. Du fait de ses ressources, il est également le seul à assurer un acheminement régulier du tissu, à travers ses structures locales et à l'attention des nouveaux membres.

\section{Rétributions matérielles et usages non partisans}

Une conséquence directe de ces nouveaux modes de distribution massive des pagnes pendant les meetings de campagne est qu'ils se retrouvent facilement en possession de femmes non militantes. Beaucoup s'y rendent par curiosité, pour écouter le programme du candidat ou pour leur dimension festive - pour profiter des performances artistiques qui les rythment -, mais également pour des raisons plus matérielles et intéressées. Chaque meeting comporte en effet un ensemble de moments très institutionnalisés où les femmes se voient remettre de l'argent par les élites partisanes présentes ${ }^{45}$. Ces dons interviennent généralement en fin de meeting et sont adressés à l'ensemble de la branche féminine locale du parti avant d'être redistribués plus tard entre les membres. Mais il est également fréquent que des sommes plus modestes soient directement offertes au moment même où elles dansent et chantent les louanges des leaders présents. Parfois, les dons peuvent également prendre la forme de distribution de nourriture. Pour beaucoup de femmes présentes, les revenus et biens matériels collectés lors des meetings politiques contribuent en partie à la subsistance du foyer ${ }^{46}$. C'est le port du pagne qui sert de sésame pour y avoir accès :

. « Atupele Floods Blantyre in Yellow », The Nation, 24 mars 2014.

44 . Entretien avec une membre de l'encadrement du PP, Blantyre, 26 octobre 2016. Il est difficile d'enquêter sur l'origine des fonds débloqués massivement par les partis au pouvoir pour leurs candidats qui reste ambiguë et sujette à de nombreuses spéculations.

45 . L. Gilman, «Purchasing Praise: Women, Dancing, and Patronage in Malawi Party Politics », Africa Today, vol. 48, n 4, 2001, p. 43-64. 


\section{DERNIÈRE VERSION AVANT PUBLICATION}

«À la fin des meetings, il y a souvent une distribution d'argent et ils donnent principalement à celles qui portent le tissu. Si vous ne le portez pas, il y a peu de chance que vous receviez de récompense ${ }^{47} »$.

Parmi ces femmes, beaucoup ne sont ni membres, ni même supportrices du parti pour lequel elles dansent, notamment en période de campagne. Une vendeuse de légume au halles de Blantyre qui se dit désintéressée de la politique partisane m'affirme ainsi posséder des pagnes de plusieurs partis et les porter en dansant et en chantant dans les meetings politiques respectifs pour avoir davantage de chances d'accéder aux élites présentes et aux cadeaux qu'elles distribuent : "Pour moi, ça n'a pas de signification, mon vote est dans mon cœur, pas sur mon $\operatorname{tissu}^{48} \gg$. Si, comme nous le détaillerons plus loin, les membres des partis dénoncent ces usages opportunistes, ils ne gênent aucunement les élites partisanes car les danses et les chants de ces femmes ne diffèrent aucunement des autres. Le revenu qu'elles obtiennent et qui justifie en grande partie leur présence s'apparente donc aussi à la rétribution d'une activité au service du parti, même si elle n'est pas vécue sur le registre du militantisme. C'est donc paradoxalement au cœur de ces événements festifs, par de nombreux aspects paradigmatiques de l'exploitation des femmes par les élites politiques, que se joue la négociation de leur participation à la compétition partisane.

\section{L'enjeu des usages détournés dans la compétition politique}

En dehors du cadre militant, les tissus investissent ensuite très vite les espaces domestiques pour être utilisés comme linge de maison, comme rideaux, ou encore dans l'espace public par les commerçantes pour transporter leurs marchandises. Ces usages utilitaires et détournés des zitenje, ainsi que l'usure avancée qu'ils entraînent, expriment un désintérêt, voire une défiance à l'égard de la politique partisane. Par exemple, Betty Maïda vendeuse de fruits au marché de Ndirande, a emballé son panier de mangues dans un pagne du PP particulièrement délavé qu'elle l'a reçu en 2014 lorsque le candidat au poste de député est venu tenir un meeting au marché où elle travaille :

Betty Maïda : Il nous a dit de venir porter le chitenje et de danser dans les meetings, mais je n'ai pas le temps d'aller aux meetings moi. Je travaille. Au début, je le portais, mais, maintenant que le tissu est usé, je l'utilise pour transporter mes marchandises.

P. G. : C'est un moyen pour vous de montrer votre affiliation au parti ?

B. T. : Non! Je n'ai rien à faire de ces histoires de politique. Ce n'est rien de plus qu'un vêtement. Je l'utilise comme tout le monde [...], ça n'a pas de signification ${ }^{49}$.

Pour elle, il n'a pas de signification identitaire ou politique mais est comparable au maïs ou à l'argent qui sont également distribués par les candidats "pour inciter les gens à voter pour eux ». Du fait de leur rapport dépolitisé au tissu, ces femmes continuent donc de l'utiliser en public même si celui-ci est très usé.

Si le fait de porter le pagne au quotidien est considéré comme un «bon usage » et un moyen de faire la publicité du parti et de ses valeurs, un tel usage purement utilitaire d'un chitenje délavé est quant à lui considéré comme dégradant pour le parti. L'enjeu est d'autant plus important pour les organisations partisanes que ces usages a priori apolitiques font l'objet d'une re-

47. Entretien avec Mary Nkholokossa, Ndirande, 23 octobre 2016.

48. Entretien avec une vendeuse de légumes aux halles de Blantyre, 24 octobre 2016.

49 . Entretien avec Betty Maïda, marché de Ndirande, 24 octobre 2016. 


\section{DERNIÈRE VERSION AVANT PUBLICATION}

politisation au service de la compétition politique. Le cas de son utilisation comme rideau, discuté par cette membre dirigeant du PP est particulièrement évocateur :

Membre dirigeant du PP : Ce n'est pas le bon usage ! [...] Après, tu trouves des photos sur Facebook et les autres partis disent que «le parti décline ».

P. G. : Que voulez-vous dire?

$\mathrm{PP}$ : Les rideaux sont très sales ! Si vous allez au village, vous verrez que les gens ne lavent jamais leurs rideaux [...]. Ils restent comme ça et finissent par s'user à cause du soleil. Les rideaux ne ressemblent à rien. Donc, quand les gens [des autres partis] voient nos tissus utilisés comme rideaux ou en guise de porte de salle de bain, ils prennent des photos et les mettent sur Facebook pour salir notre image. Surtout quand il est usé. Ils disent que l'on ne sait pas garder le contrôle sur nos biens ${ }^{50}$.

Le contrôle des usages du pagne pour s'assurer qu'ils ne donnent pas une image délavée du parti dans l'espace public présente donc un enjeu important pour les dirigeants partisans. Mon interlocutrice m'explique à ce titre que lorsque son parti était encore au gouvernement, elle avait toujours des zitenje et des T-shirts neufs dans le coffre de sa voiture : «Quand on en voyait un qui était usé dans la rue, on le changeait immédiatement ». En discutant avec les membres de l'encadrement local et national des partis, on se rend toutefois compte que leurs marges d'action sont limitées et que les utilisations privées et détournées des tissus échappent bien souvent au contrôle des partis.

Les usages domestiques et utilitaires du pagne ont débuté avec l'instauration du multipartisme. En plus de le faire acheter - ce qui avait pour effet d'en limiter les usages abusifs -, le MCP contrôlait fortement les usages de son pagne et toute utilisation non réglementaire était sévèrement réprimée par les Malawi Young Pioneers, milice armée du parti. L'instauration du multipartisme a très fortement limité la capacité des partis à contrôler les usages qui sont faits de leurs tissus. Ann Kan'gombe se désole de voir l'image de son parti ainsi dégradé sans pouvoir rien y faire :

P. G. : Si vous voyez quelqu'un faire mauvais usage du pagne, est-ce que vous faites quelque chose?

Ann Kan'gombe : Non, c'est impossible parce que, comme je le disais, les gens sont aujourd'hui libres de faire ce qu'ils veulent. C'est difficile d'aller les voir et de leur dire ça ou ça parce que vous allez immédiatement être accusés d'arrogance. On ne peut pas forcer les gens ${ }^{51}$.

Les cadres des partis font le même constat ${ }^{52}$. Une fois de plus, les organisations partisanes ne sont pas égales sur ce point. Si l'opposition n'a pas de réels moyens de pression sur les individus, le parti au pouvoir dispose d'un réseau de militants positionnés à des postes à responsabilité dans la plupart des marchés du pays (market chairmen), en charge de régler les contentieux entre commerçants, de la répartition des espaces de vente et d'autres affaires relatives à la vie du marché. Ils disposent ainsi d'un éventail de moyens de pression pour contrôler les usages qui sont faits des pagnes et rappeler à l'ordre les contrevenantes. Une vendeuse de légumes dans un marché populaire de Blantyre m'explique ainsi qu'elle utilise le tissu du PP pour transporter ses marchandises depuis que le parti n'est plus au pouvoir :

50. Entretien avec une membre de l'encadrement du PP, Blantyre, 26 octobre 2016.

51. Entretien avec Ann Kan'gombe, halles de Blantyre, 24 octobre 2016.

52 . Entretien avec la secrétaire à l'organisation de l'UDF pour le disctict de Blantyre, Ndirande, 24 octobre 


\section{DERNIÈRE VERSION AVANT PUBLICATION}

«Quand le parti perd les élections, il perd aussi la capacité de contrôler ses propriétés. Ils n'ont plus personne pour s'assurer qu'on prend soin des zitenje. Par exemple aujourd'hui, beaucoup de responsables des marchés sont des membres du DPP [le parti au pouvoir] et s'ils nous voient en faire un mauvais usage, ils peuvent vous rappeler à l'ordre. [...] Parfois aussi, le député peut venir visiter le marché. S'il vous voit utiliser leur tissu pour emballer le panier avec vos marchandises, il peut vous le confisquer et s'assurer que vous ne puissiez plus en avoir d'autres ${ }^{53} »$.

\section{Un outil personnel de participation politique pour les femmes}

Face aux nombreux usages détournés, les militantes font preuve d'une éthique militante renforcée, visible notamment dans leur utilisation publique du pagne et dans l'investissement moral qu'elles y mettent. À l'instar de toutes les militantes avec qui j'ai pu m'entretenir, Maria Nkholokossa est très critique à l'égard de ces usages utilitaires et de celles qui s'en rendent responsables :

«Le pagne est un uniforme de travail destiné à être porté. [...] Il n'est pas fait pour être utilisé comme rideaux ou comme draps. [...] Celles qui font cela ne font que salir l'image du parti. Quand vous obtenez un tissu et que vous l'utilisez pour faire des rideaux ou des draps, ça veut dire que vous ne respectez pas le parti qui est représenté ! Celles qui font ça ne s'apportent pas le respect. Ça ne fait que montrer leur pauvreté car cela montre qu'elles n'ont pas les moyens d'acheter d'autres zitenje. Alors elles utilisent ceux qu'elles reçoivent gratuitement. En plus de ça, vous allez trouver des femmes qui en ont de tous les partis et qui vont danser dans tous les meetings juste pour gagner de l'argent ${ }^{54} \gg$.

À ce rapport mercenaire et dégradant au pagne du parti, elle en oppose un usage fier et militant :

Maria Nkholokossa : La première raison pour moi, c'est pour rivaliser avec les autres partis. Quand je porte le chitenje de mon parti, c'est parce que j'espère attirer le regard des gens, les attirer pour qu'ils le rejoignent. J'ai envie que les gens admirent mon allure.

P. G. : Vous voulez dire que les gens trouvent que les tissus partisans sont beaux ?

M. N. : Pas forcément, mais ils attirent le regard et, quand les gens les voient, ils commencent à parler des développements ${ }^{55}$ qui sont pris en charge par le parti ${ }^{56}$.

Même en dehors des meetings, le port du pagne est ainsi vécu comme un moyen de participer à son niveau à la compétition politique et à la valorisation de l'image de son parti. Les pratiques vestimentaires qui lui sont associées sont donc contrôlées de manière à revendiquer une identité politique et à se distinguer des non-militantes. Face à la généralisation des usages domestiques qui usent rapidement le tissu, celles qui se considèrent comme de vraies militantes redoublent par exemple d'efforts pour le garder propre et net ; au travail, elles le portent systématiquement par-dessus un autre pagne pour pouvoir le retirer une fois assises et éviter qu'il ne s'abîme trop vite.

Pour ces femmes, l'ensemble des activités militantes dans lesquelles elles s'engagent au quotidien, comme les meetings ou les réunions, s'apparente à un travail dont il est l'uniforme.

53. Entretien avec une vendeuse de légumes, marché de Zingwangwa, 25 octobre 2016.

54. Entretien avec Mary Nkholokossa, Ndirande, 23 octobre 2016.

55 . Dans les quartiers populaires, on mesure les actions des gouvernants notamment aux

« développements » qui sont entrepris dans la localité (principalement l'aménagement de la voirie et des réseaux d'aduction, la construction de blocs sanitaires et scolaires et de ponts). 


\section{DERNIÈRE VERSION AVANT PUBLICATION}

Cela justifie à leurs yeux les rétributions matérielles qui leur sont régulièrement distribuées. Ainsi, lorsque leurs pagnes sont usés ou délavés, elles s'organisent à l'échelle de la branche locale pour en faire part aux cadres régionaux ou au membre du parlement de leur circonscription, qui sont chargés de leur en fournir de nouveaux ${ }^{57}$.

Chacune s'impose ses propres règles d'utilisation du vêtement. Pour une secrétaire d'organisation de l'UDF rencontrée au marché de Ndirande, le fait de porter le tissu au quotidien en dehors des journées de réunion partisane est un usage abusif qu'elle ne se permet pas, alors que, pour son amie présente pendant notre discussion, elle aussi membre senior de l'UDF, c'est un usage valorisant qui témoigne de sa fierté d'appartenir au parti ${ }^{58}$. Une conséquence directe est, que si les partis manquent d'outils externes pour contrôler les usages des pagnes, ceux-ci sont remplacés par un ensemble d'autocontrôles développés par les militantes elles-mêmes. Elles se reconnaissent ainsi comme les premières gardiennes des bonnes pratiques militantes.

Au-delà de la fierté associée à la participation à la vie du parti, les usages quotidiens, personnels et privés nous informent sur la dimension affective, intime et personnelle du militantisme politique. Au-delà de l'ancrage social du parti, ils donnent donc à voir les modalités de l'investissement du politique par les femmes des classes populaires.

Dans chaque entretien réalisé avec des membres des partis, le registre de l'affectif primait dans les raisons évoquées pour justifier des utilisations quotidiennes du tissu. Par exemple, depuis qu'elle a reçu son pagne en 2014, Ann Kan'gombe le porte tous les jours au marché où elle vend ses produits :

A. K. : Pour moi cela montre que j'ai beaucoup d'amour pour le parti et la bonne façon de l'utiliser c'est comme je le fais.

P. G. : Vous le faites aussi pour que les gens parlent du parti ?

A. K. : Non... quand je porte le chitenje de mon parti, les gens m'insultent... Ils me disent que la personne représentée sur ce tissu a fui le pays, qu'elle est lâche et n'est pas une bonne leader politique. Mais je m'en fiche, moi je le mets parce que j'aime le parti et que j'aime le pagne ${ }^{59}$.

Plus que de faire la promotion de l'image et des valeurs de son parti, porter le tissu au quotidien est donc un moyen d'expérimenter intimement un rapport affectif au parti. Le regard des autres sur son habit ne lui importe pas autant que la signification que ce geste revêt pour elle personnellement. Peu lui importe également si d'autres femmes portent le même tissu sans être membre du parti, tant qu'elles ne le font pas de manière dégradante, car, pour elle, l'affiliation politique et le vote finalement exprimé relèvent avant tout du domaine privé et intime.

Si l'uniforme est de rigueur afin de produire l'unité de valeur à laquelle doit s'apparenter une organisation partisane, la grande variété des façons de tailler le tissu dans des costumes parfois très sophistiqués, portés dans les meetings, est un autre indicateur de la façon dont les femmes investissent personnellement leur militantisme par l'intermédiaire du pagne. Précisons à ce titre que seules des membres des partis prennent la peine d'investir dans un costume. En dehors de leurs dérivés politiques, les tissus imprimés sont très souvent transformés en vêtements dans tous les milieux sociaux. Les coupes se sont fortement diversifiées et adaptées aux nouvelles tendances avec l'essor récent des classes moyennes et l'influence croissante de la mode

57. Ibid.. Cela est valable uniquement pour les militantes du parti au pouvoir qui possède les fonds suffisants ou pour celles de l'opposition dont le candidat a été élu dans leur localité.

58 . Entretien avec la secrétaire à l'organisation de l'UDF pour le disctict de Blantyre, Ndirande, 24 octobre 2016.

59. Entretien avec Ann Kan'gombe, halles de Blantyre, 24 octobre 2016. 


\section{DERNIÈRE VERSION AVANT PUBLICATION}

occidentale chez les jeunes générations. Les usages politiques sont dans l'ensemble moins originaux et ne s'écartent que très marginalement du modèle du national suit - une jupe longue, un haut et parfois un couvre-chef - institutionnalisé comme habit politique des femmes après l'indépendance. Toutefois, dans ces limites, les coupes peuvent varier beaucoup d'une militante à une autre en fonction des goûts et des envies. Quand elles possèdent suffisamment de tissu, des femmes de toutes origines sociales, même les plus pauvres, n'hésitent pas à dépenser des sommes importantes au regard de leurs revenus pour se faire tailler les costumes qu'elles aiment (figure 2). Le degré de raffinement avec lequel il est taillé n'est donc ni à coup sûr une preuve de la position dans la hiérarchie du parti, ni forcément un moyen de manifester un statut socioéconomique élevé, mais il est systématiquement considéré par les militantes rencontrées comme une marque de prestige et d'engagement. Le fait d'occuper une position au sein de la structure partisane, même à l'échelle très locale, est un élément important de prestige social et de respectabilité dans les quartiers populaires. Investir des sommes importantes dans un costume raffiné n'est donc pas seulement une marque d'adhésion au parti ou à son leader, mais tout autant un moyen de se faire plaisir et d'afficher publiquement son statut social ${ }^{60}$.

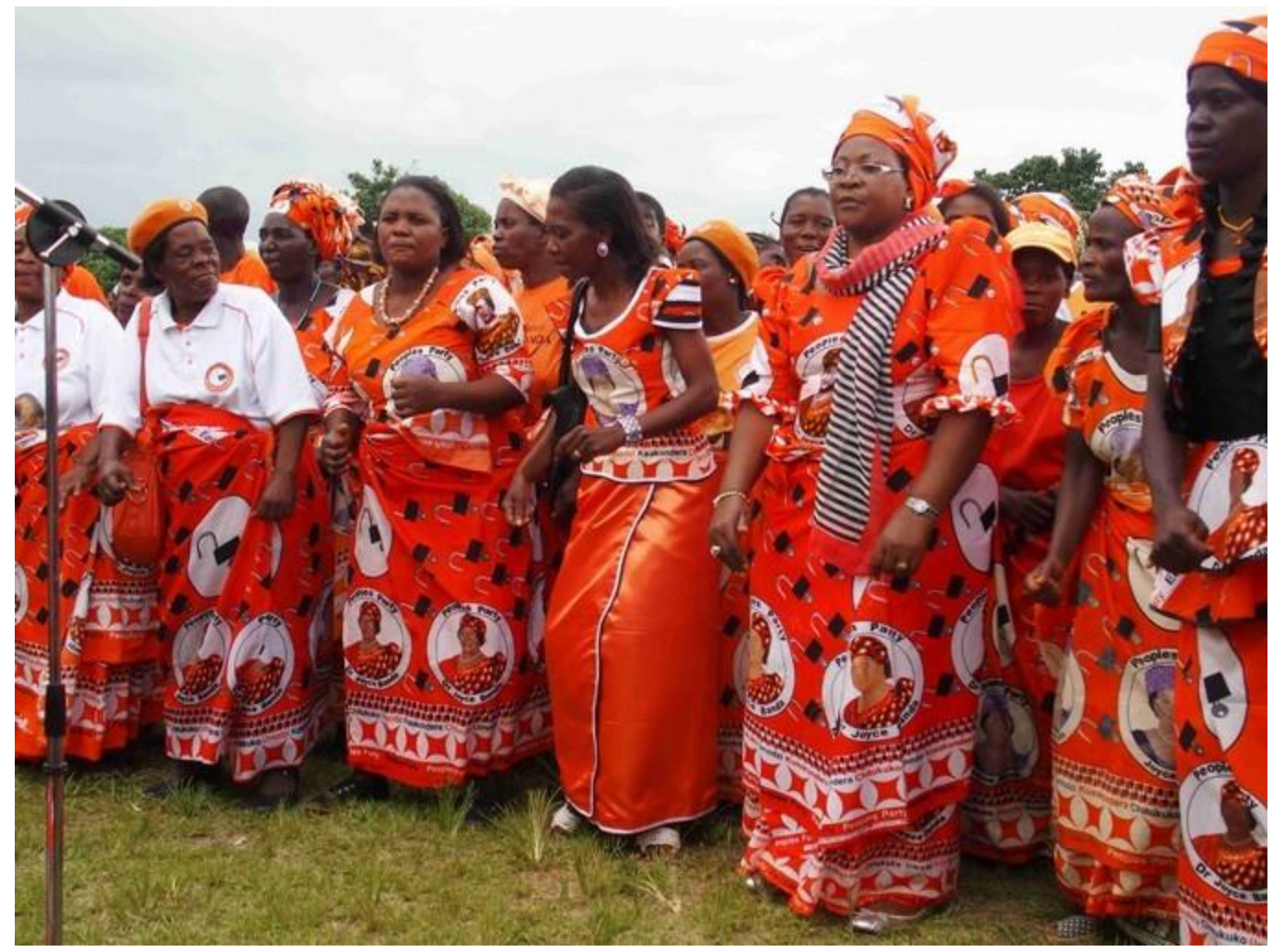

Illustration 2 : Des façons différentes de porter le vêtement du parti, meeting du PP, 16 mars 2014

\section{Conclusion}

Cet article s'est efforcé de mettre en perspective la participation politique des femmes des catégories populaires à partir des usages qu'elles font des pagnes partisans qui leur sont distribués gratuitement lors des meetings politiques. Nous avons vu que, depuis l'indépendance, le tissu imprimé aux couleurs du parti occupe une place centrale au cœur des échanges politiques verticaux et horizontaux qui structurent les organisations politiques et participent à 


\title{
DERNIÈRE VERSION AVANT PUBLICATION
}

la division sexuelle et sociale du travail militant. Il joue en cela un rôle prépondérant dans le processus de fabrique partisane. L'introduction du multipartisme et du libéralisme politique au milieu des années 1990 en a fortement transformé les conditions de distribution et élargi l'accès à des femmes qui ne soutiennent pas le parti. Les marges de manœuvre pour tailler et utiliser le pagne dans des contextes variés et en dehors des arènes politiques traditionnelles se sont étendues. Celui-ci est alors utilisé au quotidien comme un outil de participation politique et comme un moyen de négocier les modalités de leur militantisme et de leurs rapports aux élites politiques. Son utilisation domestique par des femmes non membres exprime quant à elle un rejet des partis et des schèmes de mobilisation qu'ils proposent. Toutefois, si des rapports variés au militantisme sont effectivement contenus dans les différents usages qu'elles font de leur pagne, aucune utilisation subversive visant à exprimer un mécontentement à l'égard des pratiques des élites ou du leadership du parti n'a été relevée. Le chitenje fonctionne donc davantage comme un outil d'encadrement de la participation politique des femmes et un dispositif d'achat de leur loyauté que comme une plate-forme d'expression politique utilisée par les femmes pour peser sur le processus de prise de décision.

Paul Grassin

Université Paris 1 Panthéon-Sorbonne, Centre européen de sociologie et de science politique

\begin{abstract}
Bodies as partisan infrastructures: the uses of political loincloths, parties' fabric and political participation of women of the working classes in Malawi

Political cloth showing the colors and symbols of political parties are ubiquitous in Malawian public space, during the election as well as in the daily life. It keeps circulating after political events and penetrates the domestic sphere. This paper suggest that the various uses of political cloth by party elites, party supporters and mere citizens highlights the fabric of the party as well as the conditions of poor women political participation.
\end{abstract}

